Table 1. Some parameters of cascades for uranium isotope separation (feed: natural uranium $0.725 \%{ }^{235} \mathrm{U}$; waste: $0.4 \%{ }^{235} \mathrm{U}$ )

\begin{tabular}{|c|c|c|c|}
\hline Parameter & $\begin{array}{l}\text { Gaseous } \\
\text { diffusion }\end{array}$ & Centrifugation & $\begin{array}{l}\text { Chemical and } \\
\text { jon-exchange unit }\end{array}$ \\
\hline Separation factor per stage & 1.0043 & 1.01 & 1.0213 \\
\hline \multicolumn{4}{|l|}{$3 \%{ }^{235} \mathrm{U}$} \\
\hline Number of separation stages & 951 & 309 & 193 \\
\hline $\begin{array}{l}\text { Amount of uranium in cascade } \\
\text { per unit product }\end{array}$ & 606,000 & 112,000 & 24,000 \\
\hline \multicolumn{4}{|l|}{$93 \%{ }^{235} \mathrm{U}$} \\
\hline Number of separation stages & 2777 & 1629 & 743 \\
\hline $\begin{array}{l}\text { Amount of uranium in cascade } \\
\text { per unit product }\end{array}$ & $36,993,000$ & $6,840,000$ & $1,508,000$ \\
\hline
\end{tabular}

consumption and the economic aspects still remain to be clarified [4].

Received June 1, 1976

1. Kakihana, H.: J. Chem. Soc. Japan (pure Chem.) 89, 734 (1968)

2. Shimokawa, J., Kobayshi, F.: Isotopen- praxis 6, 121 (1970); Dean, J.B. : Ger. Offen. 2155895, 25. Mai 1972

3. Ponta, A., Cǎluşaru, A : Isotopenpraxis 11 , 422 (1975)

4. Hardy, C.J.: Intern. Conf. Uranium Isotope Separation, London 1975 (Communication No. 12); Căluşaru, A.: Roumanian Patent (submitted)

\title{
A Discrete Physiological Role for Organic Colloidal Debris in Soils
}

\author{
S. Ramamoorthy \\ Dept. of Biology, University of Ottawa, Ottawa K1N 6N5, Canada \\ G.G. Leppard \\ Process Research Division, Canada Centre for Inland Waters, \\ Environment Canada, Burlington, Ontario, Canada
}

In an ultrastructural investigation of the physical relations between roots and a variety of soil particles, we observed that root-surface fibrils appeared to "invade" organic colloids. Such colloids can form a dynamic and extensive continuum in the soil and so their contact relations with root surfaces must be of great significance [1]. A monocot, Triticum aestivum, and a dicot, Daucus carota, were grown [2,3] and prepared for transmission-electron microscopy as described in [4]. Under the conditions of growth, much particulate organic matter was produced in the medium and no mineral particles were present. Particles of organic debris at the root tip were seen to be physically integrated with rhizoplane fibrils [5] at regions where ion uptake for the growing root is localized [2] and where root-surface area may be greatly extended by rhizoplane fibrils $[2,5]$.

The electron-opaque colloids seen by us to be physically "embraced" by rhizoplane fibrils were in the upper size range and thus may be considered to have a dif- fuse double layer [6] of ions. It is inferred from accessory data that these large colloids are fragments of polysaccharide gel rich in acid polysaccharide. Such large col-

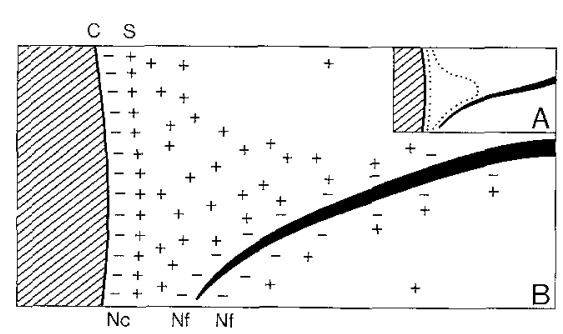

Fig. 1. The contact relations between fibril and colloid as interpreted on the basis of general physical and chemical considerations: (A) A fibril tip adjacent to the surface of a colloid. The dotted line encloses the double layer. (B) An enlarged view of the same showing the ionic environment. $C$ colloid edge, $N c$ resident negative charges on the colloid, $S$ Stern layer, $N f$ resident negative charges of the fibril. The diffuse layer is represented by the scattered positive charges between the Stern layer and the fibril loids will be plentiful in the rhizosphere as the result of natural degradation processes; it is a reasonable assumption that they have general physico-chemical features which are representative of acid polysaccharide debris found in soil.

Figure 1 represents our interpretation of the contact relations revealed by electron microscopy. Adsorbed cations give the colloid a net positive charge but remain in the soil medium by virtue of their kinetic energy. This charged layer is composed of a compact, inner, Stern layer and an outer, more extensive, diffuse layer [6] and is characterized by a zeta potential [6] upon which depends the stability of the colloid.

When the double layer is exposed to a sudden change of cation concentration such as must occur upon close approach of a fibril, there is a rapid re-establishment of adsorption equilibrium followed by redistribution of cations from the Stern layer into the diffuse layer (Fig. 1), depending on the specificity for adsorption of cations. The diffuse layer will tend to adopt the shape of a cone. As the fibril, with its carboxyl exchange sites, approaches even more closely to the colloid, its surface will interact with the curved edge of the diffuselayer cone. Thus the fibril will take a curved path as it approaches the Stern layer and acquire the cations of the outer portion of the cone by electrophoresis. When these cations move along the fibril toward the root, as the result of rootmembrane activity, they will be replenished by further capture of cations from the medium by dynamic re-establishment of the double layer of the colloid. As a result of these phenomena, colloidal organic debris bearing fixed carboxyl ligands can fulfill a discrete physiological role in soils as a scavenger of exchangeable cations for later use by roots. Cations will flow from colloid to rhizoplane fibril when the latter is extracted of cations at its proximal end by root cell membrane action. Old discarded rhizoplane fibrils could become colloidal debris for extraction by new attached ones.

Received July 25 and September 13, 1976

1. Finch, P., Hayes, M.H.B., Stacey, M., in : Soil Biochemistry, Vol. 2, p. 257 (eds. A.D. McLaren and J. Skujins). New York: Dekker 1971

2. Leppard, G.G., Ramamoorthy, S.: Can. J. Bot. 53, 1729 (1975)

3. Leppard, G.G., Colvin, J.R.: J. Cell Biol. 53, 695 (1972)

4. Leppard, G.G., et al.: ibid. 50, 63 (1971) 5. Leppard, G.G.: Science 185, 1066 (1974)

6. Wiklander, L., in: Chemistry of the Soil p. 163 (ed. F.E. Bear). New York: Van Nostrand Reinhold 1964 